\title{
Original Article \\ Burden of alcohol related admissions in the gastroenterology department of a North Indian tertiary care centre
}

\author{
Amit Soni, Rampratap Bundela, Prachis Ashdhir, \\ Rupesh Pokharna, Sandeep Nijhawan
}

ABSTRACT

Department of Gastroenterology,

Background: Alcohol has been notoriously famous since ages for many SMS Medical College, Jaipur.

ailments. Most of the gastroenterologists are confronting with an increasing number of alcohol related problems in their clinical practice. The purpose

Correspondence: Amit Soni

Email:aamitsoni@gmail.com of this study was to provide information regarding the burden of alcohol related diseases in the gastroenterology department of a tertiary care centre in Northern India.

Methods: In this hospital based study, we studied the case records of all consecutive patients admitted, between September 2014 and November 2014, in the Department of Gastroenterology, of a tertiary care center in Northern India. A total of 1038 consecutive patients ( $>18$ years) were identified. All the inpatients were categorised into either alcohol related disease or alcohol unrelated disease.

Results: Mean age of the patients was 45.59 ( \pm 14.54$)$ years. The male to female ratio was $2.4: 1$. Out of 1038 cases, 224 cases i.e. $21.5 \%$ were due to directly alcohol releated diseases. Alcohol related admission among male patients contribute to $30.4 \%$ of all the admissions. Of all the alcohol related admissions, alcoholic liver disease (ALD)accounted for the majority ( $69.6 \%$ ). Decompensated ALD formed $13.1 \%$ of the total gastroenterology inpatient workload. No female was diagnosed to have directly alcohol related disease.

Conclusion: This study provides evidence to suggest that alcohol related diseases contribute a one fifth of the in-hospital admissions in gastroenterology department.

KEYWORDS: Alcohol; alcoholic liver disease (ALD); alcohol related diseases. 


\section{Introduction}

Alcohol has been notoriously famous since ages for many ailments. According to the recent World Health Organization (WHO) report, alcohol abuse is a component cause of more than 200 diseases and injury conditions. The global situation regarding alcohol effect is dismal. Alcohol consumption results in approximately 3.3 million deaths each year, which corresponds to $5.9 \%$ of all deaths. Also, $5.1 \%$ of the global burden of disease and injury is attributable to alcohol, as measured in disability adjusted life years (DALY). The highest number of deaths are from cardiovascular diseases followed by injuries, gastrointestinal diseases (mainly liver cirrhosis) and cancers. ${ }^{1}$ There is a wide geographical variation in alcohol attributable deaths. The proportion of alcohol attributable death relative to all deaths i.e. AAFs (Alcohol Attributable Fractions) is highest in the WHO European region and lowest in African and Eastern Mediterranean regions. ${ }^{1}$

The Indian scenario is no better, rather worse. The sales of alcohol have seen a growth rate of $8 \%$ in the past three years. Chief concern today for the India is that people are beginning to drink at even younger ages. The percentage of the drinking population aged under 21 years has increased from $2 \%$ to more than $14 \%$ in last one to two decades. ${ }^{2}$ In the recent decade there have been many reports, both from India and abroad, expressing the rise in number of patients admitted to hospitals with alcohol related illnesses. ${ }^{3,4}$ In India, alcohol related problems account for more than a fifth of hospital admission, $18 \%$ of psychiatric emergencies, more than $20 \%$ of all brain injuries and $60 \%$ of all injuries reporting to emergency rooms. ${ }^{2}$ A recent study performed at our hospital revealed that alcohol is the major cause of chronic liver disease (CLD) accounting for $58 \%$ of all the CLD cases. ${ }^{5}$ This result matches with many studies from other parts of northern India. ${ }^{6,7}$ In one of a recent multicenter study from India, it was observed that alcohol was the commonest acute precipitant factor (for Acute on Chronic Liver Failure) and the commonest cause of CLD. ${ }^{6}$

Excess alcohol consumption is associated with plentiful and various medical diseases like cirrhosis, acute pancreatitis, chronic pancreatitis, cardiac diseases, head and neck cancers etc. However, the main brunt of alcohol addiction is on the liver. ${ }^{8}$ Many hypothesis have been proposed for the liver disease pathogenesis including toxic metabolite accumulation in liver, oxidative stress, mitochondrial dysfunction and hypoxia. ${ }^{9}$ Genetic factors and female gender are also well accepted risk factors. What makes alcohol related disease different from other major ailments like diabetes, coronary artery disease, is the fact that these alcohol related diseases are $100 \%$ preventable. If proper steps are taken to curb alcohol use then surely the occurrence of alcohol related diseases can be minimized if not reduced to zero.

Most of the gastroenterologists and physicians agree that with each passing year, they are confronting with an increasing number of alcohol related problems in their clinical practice. ${ }^{4}$ The purpose of this study was to provide information regarding the burden of alcohol related diseases in the gastroenterology department of a tertiary care center in Northern India.

\section{Material \& Methods}

In this hospital based study, we studied the case records of all consecutive patients admitted, between September 2014 and November 2014, in the Department of Gastroenterology, of a tertiary care center in Northern India. A total of 1038 consecutive patients ( $>18$ years) were identified. All the details of patients were collected including patients age, sex, date of admission, date of discharge, diagnosis of patient including various co morbidities. Information regarding amount of alcohol, duration of alcohol, type of alcohol consumed, CAGE questionnaire score, smoking, tobacco chewing and other addictions were also collected. Only those patients who had complete work up and a definite diagnosis made were included in the study.

All the inpatients were categorised into either alcohol related disease or alcohol unrelated disease. Patients were included in the alcohol related disease category if the disease was directly caused by alcohol (e.g. alcoholic liver disease, acute pancreatitis, chronic pancreatitis) or if the 
alcohol played a significant role in causation of the disease (e.g. post binge upper GI bleed, withdrawal syndromes including Wernicke's encephalopathy, malnutrition due to alcohol ). The definitions used are - alcohol users were defined as individuals who are currently using any of the alcohol containing products while past users were defined as those who are currently not using alcohol containing substances and who have not used such products for last one year. ${ }^{10}$

Results for continuous variables were expressed as means and standard deviation. Categorical variables were expressed as percentages. Student's t-test for continuous variables and Chi square test for discrete variables were used to test significance. A p value of less than 0.05 was considered statistically significant. SPSS21 software was used for statistical analysis.

\section{Results}

A total of 1038 consecutive patients were included in the study. Mean age of the patients was $45.59( \pm 14.54)$ years. The male to female ratio was $2.4: 1$. Out of 1038 cases, 224 cases i.e. $21.5 \%$ were due to directly alcohol related diseases. While the remaining 814 cases (78.4\%) were not directly caused by alcohol. Disease wise distribution of patient is depicted in Table 1. Alcohol related admission among male patients contribute to $30.4 \%$ of all the admissions. Of all the patients $22.9 \%$ were smoker while $32.7 \%$ were alcohol users. Among patients with alcohol related diseases, $152(67.8 \%)$ patients consumed country liquor while the remaining 72 patients $(32.1 \%)$ consumed India made foreign liquor. Whisky was the most common form of India made foreign liquor used (83.3\%) while a small proportion (16.7\%) used rum and/or beer. 164 (73.2\%) patients with alcohol related diseases scored $>2$ for CAGE questionnaire while 35 (15.6\%) had a score of $<1$ and the remaining 25 patients did not answer the questionnaire.

Association of variables (mean age, sex ratio, smoking, alcohol consumption and mean hospital stay) with directly alcohol related disease and unrelated diseases is shown in Table 2. There was no difference with respect to mean age and mean hospital stay among directly alcohol related and non alcohol related disease however history of smoking and male sex was significantly associated with alcohol related diseases ( $p$ value $<0.005$ ). The difference between male and female patient groups is shown in Table 3. Smoking and alcohol consumption was significantly associated with male sex ( $\mathrm{p}$ value $<0.005$ ).

\section{Discussion}

This study depicts the spectrum of diseases for which patients were admitted in the gastroenterology speciality, of a tertiary care center of northern India. Further, the study emphasizes on the burden being placed on the gastroenterology department due to alcohol related diseases admissions. Alcohol related diseases accounted for $21.5 \%$ of the total admissions in the gastroenterology speciality. The study also showed that extra hepatic biliary obstructive jaundice accounted for next major cause for admission in gastroenterology.

The study highlights that out of all the alcohol related admissions, alcoholic liver disease (ALD) accounted for the majority (69.6\%). Majority of these ALD patients had decompensated chronic liver disease

\section{Table 1: Disease wise distribution of patients}

\begin{tabular}{l|l} 
Diseases & $\begin{array}{l}\text { No. of Patients (\%) } \\
\text { Alcoholic Liver Disease } \\
\text { Decompensated CLD 20 } \\
\text { Alcoholic Hepatitis] }\end{array}$ \\
\hline $\begin{array}{l}\text { Acute pancreatitis (Ethanol } \\
\text { related) }\end{array}$ & $24(2.3)$ \\
\hline $\begin{array}{l}\text { Chronic Pancreatitis (Ethanol } \\
\text { related) }\end{array}$ & $44(4.2)$ \\
\hline $\begin{array}{l}\text { Extra Hepatic Biliary } \\
\text { Obstructive Jaundice }\end{array}$ & $\begin{array}{l}132(12.7) \text { [including 36 } \\
\text { CBD Stone }\end{array}$ \\
\hline $\begin{array}{l}\text { Chronic Liver Disease } \\
\text { (excluding Ethanol Related) }\end{array}$ & $108(10.4)$ \\
\hline Amoebic liver abscess & $75(7.2)$ \\
\hline $\begin{array}{l}\text { Acute Pancreatitis (excluding } \\
\text { Ethanol Related) }\end{array}$ & $\begin{array}{l}41(3.9) \text { [including 30 } \\
\text { Biliary Pancreatitis] }\end{array}$ \\
\hline Carcinoma Esophagus & $43(4.1)$ \\
\hline Koch S Abdomen & $36(3.4)$ \\
\hline NCPH & $27(2.6)$ \\
\hline Other Diseases & $352(33.9)$ \\
\hline TOTAL CASES & $1038(100)$
\end{tabular}


Table 2: Association of variables withdirectly alcoholrelated and alcohol unrelated diseases

\begin{tabular}{|c|c|c|c|c|}
\hline \multirow{2}{*}{ S. No } & \multirow{2}{*}{ Variables } & \multicolumn{2}{|c|}{ Gastroenterology Cases } & \multirow[t]{2}{*}{$\begin{array}{l}\text { Level of significance } \\
\text { P value }\end{array}$} \\
\hline & & $\begin{array}{l}\text { Directly Alcohol related } \\
(\mathrm{n}=224)\end{array}$ & $\begin{array}{l}\text { Non alcohol related } \\
(\mathrm{n}=814)\end{array}$ & \\
\hline 1 & Mean Age (SD), years & $44.09(11.06)$ & $46.00(15.32)$ & 0.084 \\
\hline 2 & Sex Ratio (F/M) & $0 / 224$ & $303 / 511$ & $\leq 0.005$ \\
\hline 3 & H/o Smoking (n) & $97(43.3 \%)$ & $143(17.5 \%)$ & $\leq 0.005$ \\
\hline 4 & Alcohol consumption (n) & $224(100 \%)$ & $121(14.8 \%)$ & $\leq 0.005$ \\
\hline 5 & Mean Hospital stay, days & $5.03( \pm 2.72)$ & $4.58( \pm 2.47)$ & 0.019 \\
\hline
\end{tabular}

Table 3: Distribution of case as per sex

\begin{tabular}{l|l|l|l|l}
\multirow{2}{*}{ S. No } & \multirow{2}{*}{ Variables } & \multicolumn{2}{|c|}{ Total Cases } & \multicolumn{2}{|l}{$\begin{array}{l}\text { Level of significance } \\
\text { P value }\end{array}$} \\
\cline { 3 - 5 } & & Male $(\mathbf{n}=\mathbf{7 3 5})$ & Female $(\mathbf{n}=\mathbf{3 0 3})$ & \\
\hline $\mathbf{3}$ & Mean Age (SD), years & $44.73( \pm 13.95)$ & $47.69( \pm 15.69)$ & $\leq 0.005$ \\
\hline 4 & H/o Smoking & $226(30.7 \%)$ & $11(3.6 \%)$ & $\leq 0.005$ \\
\hline 5 & Alcohol consumption & $345(46.9 \%)$ & $0(0 \%)$ & $\leq 0.005$ \\
\hline
\end{tabular}

(87.17\%) along with complications like ascites, GI bleed, hepatic encephalopathy and/or spontaneous bacterial peritonitis. Decompensated ALD formed 13.1\% of the total gastroenterology inpatient workload. Hislop et al studied gastroenterology in patient workload due to alcohol related illness. They concluded that $44 \%$ of gastroenterology in patients were due to alcohol related diseases. In patients with decompensated ALD formed $37 \%$ of gastroenterology workload. ${ }^{11}$

This proves that alcohol works as a double edged sword. It not only cause morbidity and mortality among individuals but also consumes resources needed to tackle these serious ailments and their complications. The results also communicates the high occurrence of alcohol related disease among males (30.4\%) despite female gender being a well known risk factor for ALD. This could well be explained by the fact that alcohol consumption is still a taboo for women in majority of India. Not even a single female was diagnosed to have directly alcohol related disease in our report. A study by Wadell et al, also concluded that there is an increased prevalence of alcohol related diseases among males. ${ }^{4}$ An epidemiological study by Sundaram et al conducted in a rural area of Rajasthan also arrived at the result that alcohol consumption is still infrequent among women. ${ }^{12}$

The study demonstrates that gastroenterology inpatient practice is now dominated by the treatment of chronic liver disease, particularly among males. There were relatively few inpatients with Non Cirrhotic Portal Hypertension. Country liquor was the most frequent type of alcohol beverage being used. Alcohol was the main etiological factor for CLD. A new study from India, demonstrated that CLD due to alcohol has a significant rising trend and it further showed that since 2007, alcoholic liver disease has been the commonest cause of CLD and almost three fourths of these patients have decompensated disease at presentation. ${ }^{3}$

Alcohol consumption was seen in $32.7 \%$ of all the inpatients in our hospital. Studies from various regions of northern India have shown the 1 year prevalence of alcohol use to be between $25 \%$ and $40 \%{ }^{13,14}$ In a study a tertiary care centrein Delhi, alcohol use was found to be $21.6 \%$ among all hospital inpatients, while the figure rose to $31 \%$ when calculated exclusively among males..$^{10}$ Our 
data matches very closely to the alcohol user percentage in this Delhi based study. Smoking was seen in a high percentage of patients with alcohol related disease (43.3\%) when compared to alcohol unrelated diseases (17.5\%). Thus, emphasizing that patients with alcohol use have higher propensity for addiction with smoking. There was no statistically significant difference in the mean age and mean hospital stay when patients group with alcohol related and unrelated diseases were compared. In view of people consuming country liquor predominantly, which contains variable percentage of alcohol (20-50\%), the amount of alcohol consumed was not calculated in this study. None of the patient was admitted solely for acute ethanol intoxication, withdrawal syndrome or malnutrition due to alcohol. This may be explained by the tendency for patients with these problems to get admitted in other departments like medicine and psychiatry.

This result focus on the need for a team approach among gastroenterologists, physicians and psychiatrists to treat the ongoing menace of alcohol related disease followed by de addiction. The measures will be inadequate if nothing is done to prevent the public from becoming an alcohol addict. The current prevalence pattern of alcohol related diseases requires urgent attention of doctors, para medicals, government and non government organizations (NGOs). Multiple factors such as poverty, illiteracy, ignorance, peer group influence and easy alcohol availability play a role in making a person addicted to alcohol. Due to these numerous factors involved, a curb on alcohol use becomes very difficult. Even the resources needed to treat and further prevent alcohol use would be enormous. Understanding on alcohol abuse will help to device/design better preventive and treatment measures.

This study is first of its kind from India wherein the burden of alcohol related disease has been measured in gastroenterology department. This study has many limitations including less precise data collection due to retrospective nature and short duration study period. Also, since this is a hospital based study so the study group might not be the true representative of the whole population. Lastly, patients might not have accurately reported the quantity and frequency of alcohol consumption.

\section{Conclusion}

This study provides evidence to suggest that alcohol related diseases contribute a major share to the in-hospital admissions in gastroenterology department. The results emphasize on holding strict strategies to curb the growing alcohol abuse pandemic.

\section{References}

1. World Health Organization. Global status report on alcohol and health. 2014.P 45-5-7

2. Prasad R. Alcohol use on the rise in India. Lancet. 2009;373:17-8.

3. Ray G. Trends of Chronic Liver Disease in a Tertiary Care Referral Hospital in Eastern India. Indian J Public Health. 2014;58:186-94.

4. Waddell TS, Hislop WS. Analysis of Alcohol-Related Admissions in Gastroenterology, Cardiology and Respiratory Medicine. Scott Med J. 2003;48:114-6

5. Jhajharia A, Soni A, Pokharna Ret al.Spectrum of chronic liver disease admitted to a medical college hospital in northern India: is there cause for concern? Indian $J$ Gastroenterol. 2014;33:480-1.

6. Shalimar, Saraswat V, Singh S et al. Acute on chronic liver failure in India: the INASL Consortium experience. J Gastroenterol Hepatol. 2016;31(10):1742-1749.

7. Shalimar, Kumar D, Vadiraja PK et al. Acute on chronic liver failure because of acute hepatic insults: Etiologies, course, extrahepatic organ failure and predictors of mortality. J Gastroenterol Hepatol. 2016;31:856-64.

8. Shield K. D, Parry C, Rehm J. Chronic diseases and conditions related to alcohol use. Alcohol Research: Current Reviews. 2013;35:155-173.

9. Seth D, Haber PS, Syn WK, Diehl AM, Day CP. Pathogenesis of alcohol-induced liver disease: classical concepts andrecent advances. $J$ Gastroenterol Hepatol. 2011;26:1089-1105

10. Thilakan VC, Rasania SK. Alcohol use among in-patients of a medical college hospital in Delhi. Indian J Psychiatry. 2013;55:405-7.

11. Hislop WS, Heading RC. Impact of Alcohol related disease and inpatient Workload of Gastroenterologists in Scotland. Scott Med J. 2004;49:57-60.

12. Sundaram KR, Mohan D, Advani GB, Sharma HK, Bajaj JS. Alcohol abuse in a rural community in India. Part 
I: Epidemiological study. Drug Alcohol Dependence. 1984;14:27-36.

13. Mohan D, Sharma HK, Darshan S, Sundaram KR, Neki JS. Prevalence of drug abuse in young rural males in Punjab.
Indian J Med Res. 1978;68:689-94.

14. Varma VK, Singh A, Singh S, Malhotra A. Extent and pattern of alcohol use and alcohol related problems in North India. Indian J Psychiatr. 1980;22:331-7. 